\title{
ZigBee-based Sensor Network for Indoor Location and Tracking Applications
}

\author{
Y. Alvarez, Senior Member, IEEE and F. Las Heras, Senior Member, IEEE
}

\begin{abstract}
Practical asset and people tracking in indoor scenarios using Received Signal Strength (RSS) ZigBee-based sensor network is presented. The Indoor Location System (ILS) is based on a novel algorithm that uses relative field levels gathered by a set of transmitter nodes. These levels are introduced into a cost function which minimum gives the asset location estimation. The use of relative field levels-based algorithm avoids the need of system calibration due to signal strength fluctuation. The method is tested in two real indoor scenarios: the first example is devoted to evaluate the influence of the number of transmitting nodes on the position estimation accuracy as well as the number of samples to be averaged aiming to reduce uncertainty. The second example shows a realistic case of asset tracking, also studying the influence of sample averaging and number of transmitting nodes.
\end{abstract}

Keywords - wireless sensor networks, indoor location systems, received signal strength, ZigBee, radiodetermination.

\section{INTRODUCCION}

$\mathrm{E}^{\mathrm{N}}$ los últimos años, los Sistemas de Localización en Interiores (Indoor Location Systems, ILS) han sido objeto de estudio debido a su amplio abanico de aplicación para localización, seguimiento de objetos y personas. Dichos sistemas se han desarrollado en gran medida como complemento a los sistemas de navegación global por satélite [1], tales como GPS (Global Positioning System), que ofrecen precisiones de localización en exteriores entre $1-10 \mathrm{~m}$, pero incapaces de funcionar en interiores al no tener línea de visión directa con la constelación de satélites.

La localización y seguimiento en interiores requiere el desarrollo de técnicas que permitan mitigar los siguientes problemas: en primer lugar, se trata de escenarios con elevado multicamino, debido a la reflexión de la señal en obstáculos (paredes, suelos, mobiliario). Asimismo, no siempre es posible disponer de línea de visión directa entre los elementos que conforman el ILS, de forma que la señal puede sufrir elevada atenuación si los elementos están emplazados en diferentes estancias [2-4]. Finalmente, el escenario de despliegue del ILS puede ser cambiante, con lo que no es posible emplear un modelo preciso para la caracterización de los efectos mencionados de multicamino y atenuación.

Se han propuesto diferentes tecnologías para su implementación de los ILS, tales como infrarrojos, ultrasonidos, y sistemas basados en radiofrecuencia, tal y

Y. Alvarez, Área de Teoría de la Señal y Comunicaciones, Universidad de Oviedo, Campus Universitario de Gijón, 33203, Gijón (Asturias), España. alvarezyuri@uniovi.es

F. Las Heras, Área de Teoría de la Señal y Comunicaciones, Universidad de Oviedo, Campus Universitario de Gijón, 33203, Gijón (Asturias), España. flasheras@uniovi.es

Corresponding autor: Yuri Alvarez como se resume en la Tabla 3 de [5]. Estos últimos pueden clasificarse en dos grandes grupos: por una parte, aquellos basados en el tiempo de vuelo (Time-of-Fly, ToF) [6], donde el cálculo de la posición se estima a partir del retardo de propagación de la señal entre un transmisor y un receptor; y por otra parte, los sistemas basados en nivel de señal recibido (Received Signal Strength, RSS), en los que la distancia se estima a partir de la atenuación experimentada por la señal al propagarse [5].

La precisión de los ILS que emplean RSS depende en gran medida del modelo empleado para caracterizar la propagación de la señal. Por ejemplo, algunos sistemas emplean la técnica de fingerprinting consistente en crear una base de datos con medidas tomadas en el escenario donde se despliega el ILS $[5,7]$. A continuación, se comparan las medidas recibidas del objeto o persona a monitorizar con las almacenadas en la base de datos. Este sistema permite establecer un modelo preciso del escenario al tener en cuenta todos los efectos de propagación en interiores, pero como contrapartida requiere la creación de la base de datos, la cual dejaría de tener validez si el escenario donde se despliega el ILS sufre modificaciones.

Otros modelos para RSS se basan en asumir unas condiciones de propagación en espacio libre con presencia de multicamino. Este modelo teórico puede ajustarse con medidas en el escenario de despliegue del ILS para ofrecer una mayor precisión.

Se han propuesto diferentes tecnologías de soporte para la implementación de ILS basados en RSS, debido a la sencillez de su implementación, dado que sólo es necesario disponer de dispositivos capaces de proporcionar información de nivel de señal recibida. Entre estas tecnologías, las más extendidas son ZigBee [7-11], RFID [12,13], Bluetooth [14] o WiFi [15]. En el caso de WiFi y Bluetooth, presentan la ventaja de que estas tecnologías se encuentran disponibles en muchos dispositivos móviles, así como su elevada penetración entre los usuarios. RFID se ha popularizado en los últimos años por el bajo coste de las etiquetas pasivas, empleándose para aplicaciones de corto alcance. En el caso de ZigBee, si bien su mercado se limita fundamentalmente a redes de sensores de carácter industrial y domótico, los nodos basados en esta tecnología tienen niveles de consumo de energía significativamente menores que WiFi, RFID, y Bluetooth.

Dentro del amplio abanico de ILS basados en RSS, algunos sistemas han sido evaluados e implementados satisfactoriamente para la localización, seguimiento de personas y objetos, tales como Maximum Likelihood Estimator (MLE) [9], Ecolocation [16], y MoteTrack [17].

En general, el diseño e implementación de los sistemas ILS está sujeto a las siguientes condiciones: i) extensión del 
escenario de despliegue, ii) limitaciones para el emplazamiento de los nodos del ILS, iii) especificación de la precisión requerida, y iv) el número máximo de objetos o personas a monitorizar simultáneamente. La precisión dependerá en gran parte de la densidad de nodos del ILS, de forma que si el usuario especifica la precisión mínima, es posible determinar el número mínimo de nodos requerido. En la práctica, se indicará la precisión del ILS acompañada de un valor de fiabilidad (por ejemplo, se conseguirá una precisión de al menos $1 \mathrm{~m}$ en el $90 \%$ de las veces).

La presente contribución describe un ILS basado en RSS implementado sobre la tecnología ZigBee. En trabajos previos $[18,19]$ se llevó a cabo la caracterización de la propagación en interiores, para evaluar la precisión del modelo de propagación empleado en el ILS. En esta contribución se pretende demostrar la viabilidad de dicho sistema ILS para localización, seguimiento de personas y objetos. Asimismo, se ha mejorado el algoritmo de procesado para evitar la necesidad de una etapa previa de calibración [19].

\section{ALGORITMO RSS}

En los ILS basados en RSS, una forma de convertir el nivel de atenuación de la señal electromagnética en distancia se puede realizar a partir de la ecuación de propagación en espacio libre [20]:

$$
E_{\text {eval }}=E_{\text {ref }}(\lambda / 4 \pi R)
$$

Donde $\lambda$ es la longitud de onda, $E_{\text {eval }}$ es el nivel de campo evaluado en el nodo emplazado en el objeto o persona a localizar (a este nodo se le denominará nodo repetidor). $E_{\text {ref }}$ es un nivel de referencia de campo, habitualmente evaluado en los nodos estáticos o nodos transmisores, cuya posición es conocida.

Considerando que los nodos estáticos y el nodo repetidor están emplazados en las posiciones $\left(x^{\prime}, y^{\prime}\right)$ y $(x, y)$ respectivamente, y bajo la suposición de que $z ; z$, la distancia entre los nodos viene dada por:

$$
R=\left(\left(x-x^{\prime}\right)^{2}+\left(y-y^{\prime}\right)^{2}\right)^{1 / 2}
$$

Como se ha comentado en la sección I. Introducción, el modelo de propagación en espacio libre puede no ser suficientemente preciso debido a que el multicamino puede provocar una atenuación del campo más rápida que $1 / R$. Es por ello que se ha propuesto mejorar el modelo de propagación considerando términos de atenuación de orden cuadrático y cúbico [21]:

$$
\begin{aligned}
E_{\mathrm{med}} & =E_{\mathrm{ref}}\left((\lambda / 4 \pi R)+\left(\lambda^{2} / 8 \pi^{2} R^{2}\right)\right. \\
& \left.+\left(\lambda^{3} / 16 \pi^{3} R^{3}\right)\right)
\end{aligned}
$$

En el modelo de propagación (3) es posible añadir otros términos, tales como la atenuación adicional que sufre la señal al atravesar paredes [2,3], asumiendo que se conoce la distribución del escenario donde se despliega el ILS.

La resolución del problema de localización pretende determinar las coordenadas $\left(x^{\prime}, y^{\prime}\right)$ de la radiobaliza o nodo emplazado en la persona u objeto a localizar a partir de los valores RSS medidos en un conjunto de $N$ nodos estáticos cuya posición $\left(x_{\mathrm{n}}, y_{\mathrm{n}}\right)$ es conocida.

El cálculo de las coordenadas $\left(x^{\prime}, y^{\prime}\right)$ se puede realizar mediante la minimización de una función de coste (4), $f_{\text {cost ant }}{ }^{\left(x^{\prime}, y^{\prime}\right)}$, [19] que relaciona el campo medido en cada uno de los $N$ nodos estáticos, con el campo evaluado empleando la expresión (3).

$$
f_{\text {cost_ant }}\left(\mathrm{x}^{\prime}, \mathrm{y}^{\prime}\right)=\sum_{n=1: N} \mid E_{\mathrm{eval}, \mathrm{n}}\left(^{\left(y^{\prime}, y^{\prime}\right)}-\left.E_{\text {med,n }}\left(x^{\prime}, y^{\prime}\right)\right|^{2}\right.
$$

Donde $E_{\text {eval,n }}\left(x^{\prime}, y^{\prime}\right)$ es el nivel de campo debido al nodo estático $n$-ésimo, evaluado en el punto $\left(x^{\prime}, y^{\prime}\right)$ mediante la expresión (3), y $E_{\text {med,n }}{ }^{\left(x^{\prime}, y^{\prime}\right)}$ es el nivel de campo medido.

Si se discretiza el escenario bajo estudio en un conjunto de puntos $\left(x^{\prime}, y^{\prime}\right)$, es posible evaluar en cada punto dicha función de coste. Aquella posición que proporcione el valor más bajo de la función de coste será la que se corresponda con la mejor estimación de la posición del objeto o persona a localizar. Las capacidades de cálculo de un ordenador portátil convencional permiten evaluar miles de posiciones en menos de un segundo, de forma que el tiempo de procesado asociado a la resolución del problema no ralentiza el sistema de localización.

La función de coste (4) presenta el inconveniente de que relaciona niveles absolutos de campo. Por ello, es necesario caracterizar primero el valor $E_{\text {ref, }}$ el cual dependerá del tipo de dispositivo empleado (nodos ZigBee, balizas Bluetooth, etc.).

Para evitar la necesidad de tener que calibrar el sistema (es decir, calcular $E_{\text {ref), }}$ en esta contribución se propone una modificación de la función de coste (4) que emplea niveles de señal relativos. La idea se ilustra en la Fig. 1: en primer lugar, se calculan las $\mathrm{M}$ combinaciones de diferencias de niveles de campo evaluado y medido entre pares de nodos estáticos (5):

$$
\begin{aligned}
& \Delta_{\mathrm{eval}, \mathrm{m}}\left(x^{\prime}, y^{\prime}\right)=\left|E_{\mathrm{eval}, p}\left(x^{\prime}, y^{\prime}\right)-E_{\mathrm{eval}, q} q^{\left(x^{\prime}, y^{\prime}\right)}\right|,
\end{aligned}
$$

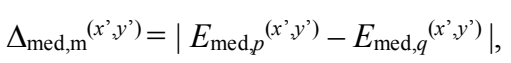

$$
\begin{aligned}
& p=[1,2, \ldots, N-1] ; \text { para cada } p: q=[p+1, \ldots, N] \text {, } \\
& m=[1,2, \ldots, M], \operatorname{con} M=N(N-1) / 2
\end{aligned}
$$

A continuación se establece una nueva función de coste que relacione las diferencias de niveles de campo evaluado y medido:

$$
\begin{aligned}
& f_{\operatorname{cost}}\left(x^{\prime}, y^{\prime}\right)=\sum_{m=1: M}\left|\Delta_{\text {eval }, m}\left(x^{\prime}, y^{\prime}\right)-\Delta_{\text {med }, m}\left(x^{\prime}, y^{\prime}\right)\right|^{2} \\
& M=N(N-1) / 2
\end{aligned}
$$

Al igual que en (4), para cada punto de evaluación ( $\left.x^{\prime}, y^{\prime}\right)$ se obtendrá un valor de la función de coste, $f_{\operatorname{cost}}\left(x^{\prime}, y^{\prime}\right)$. Aquel punto donde, para un conjunto de valores RSS de entrada se obtenga el mínimo de la función de coste es donde se asumirá que se encuentra el objeto o persona a localizar.

El hecho de emplear diferencias de niveles de campo permite eliminar errores sistemáticos que afectan a todos los nodos estáticos, como puede ser la ligera caída del nivel de señal emitido a medida que se va agotando la batería de los nodos. La contrapartida es un aumento de la carga computacional proporcional al cuadrado del número de nodos 
de la red $(N(N-1) / 2)$. No obstante, en pruebas realizadas con un ordenador portátil y con $N=6$ nodos, el tiempo de evaluación de la función de coste (6) en un conjunto de miles de puntos $\left(x^{\prime}, y^{\prime}\right)$ ha sido inferior a un segundo. Por tanto, dicho incremento del tiempo de cálculo no es significativo en la práctica, teniendo un impacto despreciable en el funcionamiento del sistema.
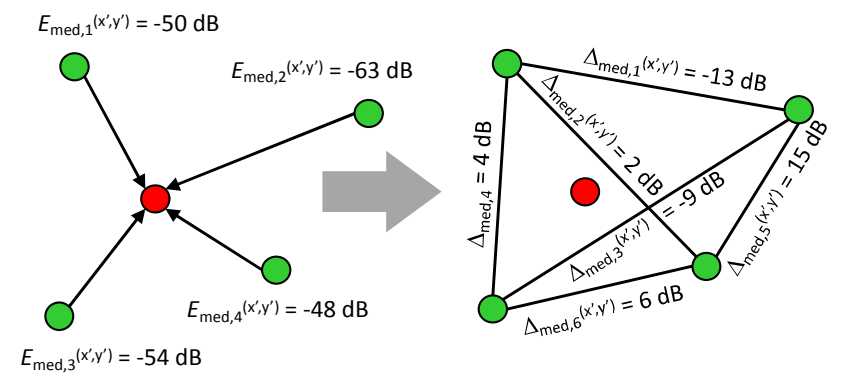

Figura 1. Concepto de ILS basado en RSS empleando niveles de señal absolutos (izquierda) y relativos (derecha).

\section{IMPLEMENTACIÓN DE LA RED DE SENSORES}

Dentro de las diferentes tecnologías para la implementación de la infraestructura del ILS, en la presente contribución se han utilizado nodos ZigBee en la banda de $2.45 \mathrm{GHz}$ [22,23], debido a su coste, nivel de estandarización, así como la sencillez para la reconfigurabilidad de la red, pudiendo modificar el modo de funcionamiento de un nodo fácilmente mediante software.

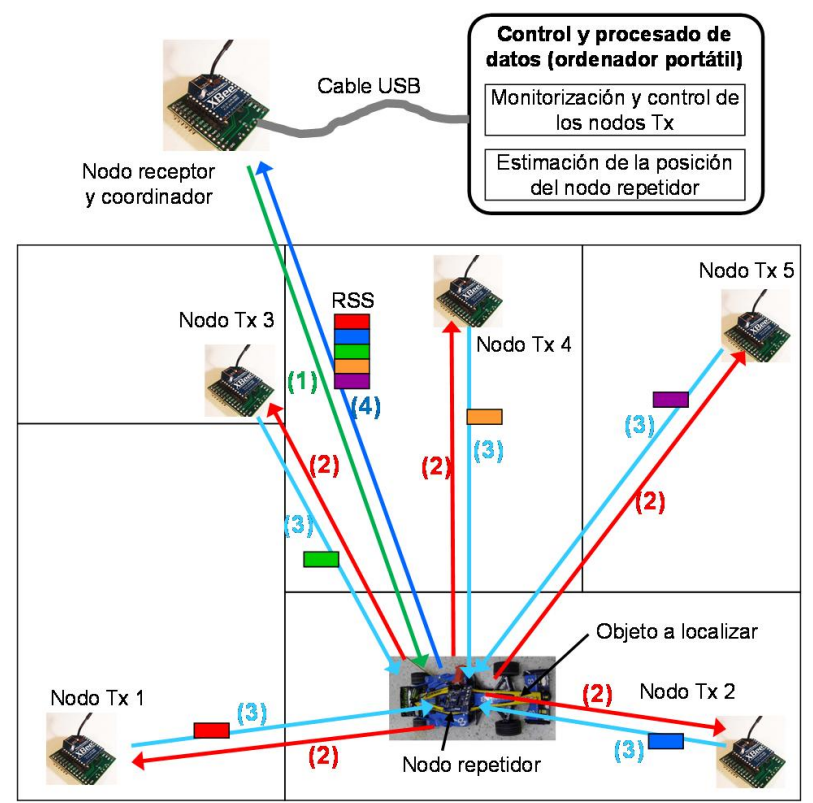

Figura 2. Implementación del ILS basado en RSS empleando nodos ZigBee. Los números indican la secuencia de comunicación para adquirir la información de RSS (representada mediante rectángulos).

Dentro de la gama de nodos ZigBee se han elegido aquellos capaces de proporcionar el nivel de RSS para cada trama recibida [24]. Dichos nodos soportan una tasa de trasferencia de $250 \mathrm{kbps}$, lo que permite el empleo de la red para otras funciones en paralelo, como por ejemplo, control domótico, envío de datos de temperatura, humedad, etc. mediante el conexionado de los correspondientes sensores a los nodos estáticos.

La topología de la red de sensores ZigBee se muestra en la Fig. 2. Básicamente se tienen tres tipos de nodos: i) un conjunto de $N$ nodos estáticos o transmisores, emplazados en posiciones conocidas $\left(x_{\mathrm{n}}, y_{\mathrm{n}}\right)$. ii) Un nodo repetidor emplazado en el objeto a persona a localizar (por ejemplo, un vehículo por control remoto). iii) Un nodo receptor y coordinador de la red, que hará de interfaz entre el dispositivo encargado de procesar los datos y calcular la posición, y la red de sensores ZigBee.

El modo de operación es el siguiente (indicado con números en la Fig. 2): 1) el coordinador, a través del nodo repetidor 2), despertará periódicamente a los nodos estáticos. Éstos medirán el nivel de señal recibido de la petición, la cual la enviarán encapsulada en la trama de respuesta. Dicha respuesta pasará nuevamente por el nodo repetidor 3 ), que la reenviará al nodo receptor/coordinador 4). La información RSS se enviará al equipo que ejecuta el algoritmo de localización, para el cálculo y representación de la posición estimada.

Un parámetro importante en el diseño del ILS es el consumo de energía, ya que los nodos estáticos pueden estar emplazados en ubicaciones de difícil acceso. Para los nodos ZigBee elegidos, la Fig. 3 representa la duración de la batería frente al tiempo que los nodos permanecen dormidos, teniendo en cuenta las especificaciones de fabricante y una alimentación con una batería alcalina AA de $2400 \mathrm{mAh}$ [25].

Se ha elegido una tasa de muestreo de $500 \mathrm{~ms}$ como compromiso entre tiempo de actualización de la posición del objeto a localizar y duración de la batería. De esta forma el nodo permanecerá dormido $490 \mathrm{~ms}$ (ciclo de trabajo del $2 \%$ ). Como se observa en la Fig. 3, el tiempo de vida de las baterías sería de unos 3 meses.

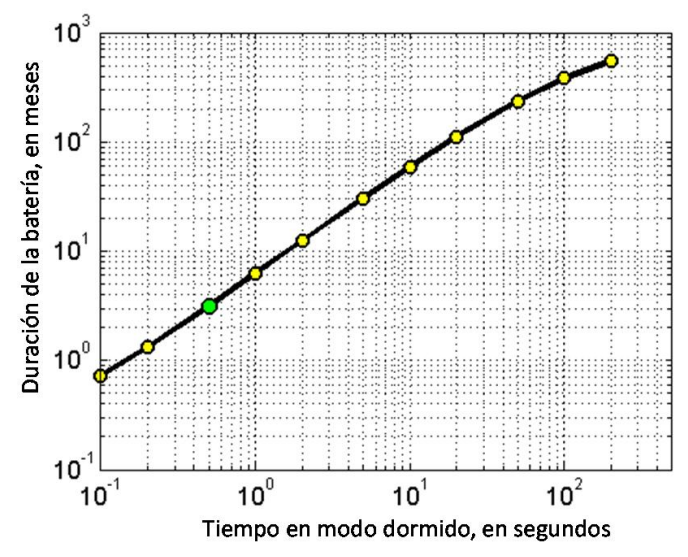

Figura 3. Duración de la batería vs. tiempo en modo dormido de un nodo ZigBee. Tiempo activo: $10 \mathrm{~ms}$ en todos los casos. Batería alcalina de 2400 $\mathrm{mAh}$ tipo AA. El punto verde representa el punto de trabajo del nodo empleado en esta contribución.

\section{ANÁLISIS DE LA PRECISIÓN DEL SISTEMA ILS}

Una vez descrito el ILS, en esta sección se analizará la precisión del mismo así como la incertidumbre en la posición en función de parámetros tales como el número de nodos 
estáticos de la red, y el promediado de las medidas de RSS.

Como escenario se ha considerado una vivienda de $65 \mathrm{~m}^{2}$ cuyo plano se ha representado en la Fig. 4. La posición de los nodos estáticos $(N=6)$ se ha realizado teniendo en cuenta la distribución del mobiliario en cada habitación con el fin de lograr el menor bloqueo de la señal emitida por los nodos estáticos, tratando de maximizar el área de cobertura. En todos los casos, los nodos están ubicados a aproximadamente $1.5 \mathrm{~m}$ de altura.

Se han seleccionado 5 posiciones aleatorias dentro de la vivienda, indicadas en la Tabla I. En cada posición se adquirirán 100 medidas de RSS. Dado que el tiempo de adquisición es de $50 \mathrm{~s}$ por posición, se consideró oportuno estudiar la influencia de la variabilidad del escenario, de forma que durante la toma de medidas se solicitó a una persona que se moviera arbitrariamente por la vivienda.

TABLA I

POSICIONES DE EVALUACIÓN DEL SISTEMA ILS

\begin{tabular}{cccccc}
\hline \hline & POS. \#1 & POS. \#2 & POS. \#3 & POS. \#4 & POS. \#5 \\
\hline$x^{\prime}$ & $6.5 \mathrm{~m}$ & $9.3 \mathrm{~m}$ & $5.8 \mathrm{~m}$ & $3.5 \mathrm{~m}$ & $1.7 \mathrm{~m}$ \\
$y^{\prime}$ & $1.5 \mathrm{~m}$ & $5.7 \mathrm{~m}$ & $5.5 \mathrm{~m}$ & $4.5 \mathrm{~m}$ & $1.5 \mathrm{~m}$ \\
\hline \hline
\end{tabular}

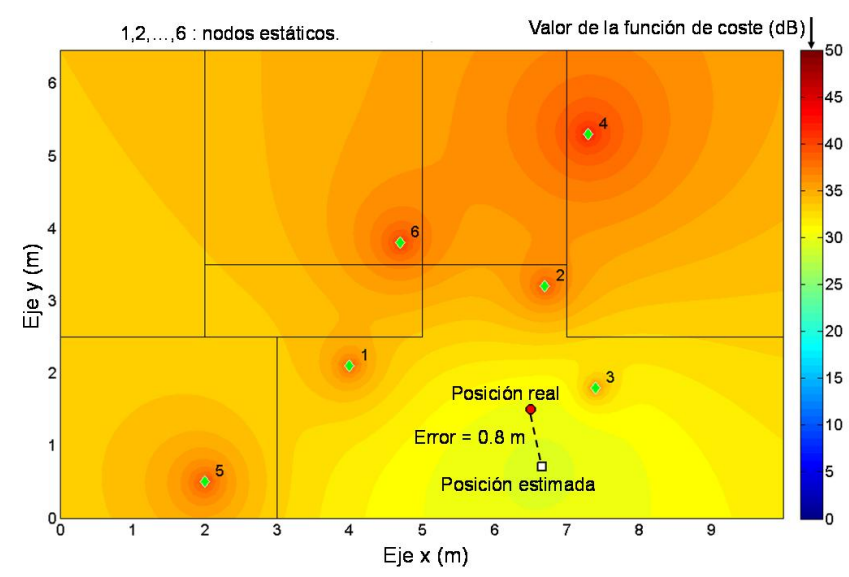

Figura 4. Estimación de la posición empleando el ILS basado en RSS Posición de evaluación \#1 (punto rojo: posición del nodo repetidor). Los puntos verdes indican la posición de los nodos estáticos. El punto blanco indica la posición estimada.

En primer lugar se analizan los valores de RSS cuando el nodo repetidor se halla emplazado en la Pos. \#1 (punto rojo en la Fig. 4). Los valores RSS adquiridos para cada uno de los 6 nodos estáticos se representan en la Fig. 5, donde puede observarse que la señal medida es bastante estable, concluyéndose que el movimiento de la persona por la casa no ha tenido una repercusión significativa en las medidas. Como sería esperable, el mayor nivel de RSS corresponde al nodo estático 3, que es el más próximo a la ubicación \#1.

Es necesario indicar que el algoritmo tiene en cuenta la atenuación de las paredes, añadiendo $3 \mathrm{~dB}$ de pérdidas [2,3] en el modelo de propagación (3) por cada pared existente entre el nodo estático $\left(x_{\mathrm{n}}, y_{\mathrm{n}}\right)$ y la posición de evaluación $\left(x^{\prime}, y^{\prime}\right)$.

Una vez estudiados los niveles de RSS, se procede al cálculo de la posición del nodo repetidor. Para ello, se discretiza la vivienda en una malla de puntos separados $20 \mathrm{~cm}$ en $x$ y en $y$, resultando en 51 x $33=1683$ pares $\left(x^{\prime}, y^{\prime}\right)$ donde la función de coste (6) es evaluada. El valor de la función de coste en cada punto se ha representado en la Fig. 4. El mínimo corresponde a la posición $\left(x^{\prime}, y^{\prime}\right)=(6.6,0.7) \mathrm{m}$, de forma que el error cometido es de $0.8 \mathrm{~m}$. Nótese que el error de posicionamiento se define como la distancia euclídea entre la posición real del nodo repetidor (definidas en la Tabla I) y la posición estimada por ILS a partir de las medidas de RSS.

Los resultados mostrados en la Fig. 4 corresponden al promediado de los 100 valores de RSS. Sin embargo, un sistema ILS que necesite $50 \mathrm{~s}$ para determinar la posición no es viable, por lo que es necesario analizar la relación entre el error cometido y el número de muestras promediadas.

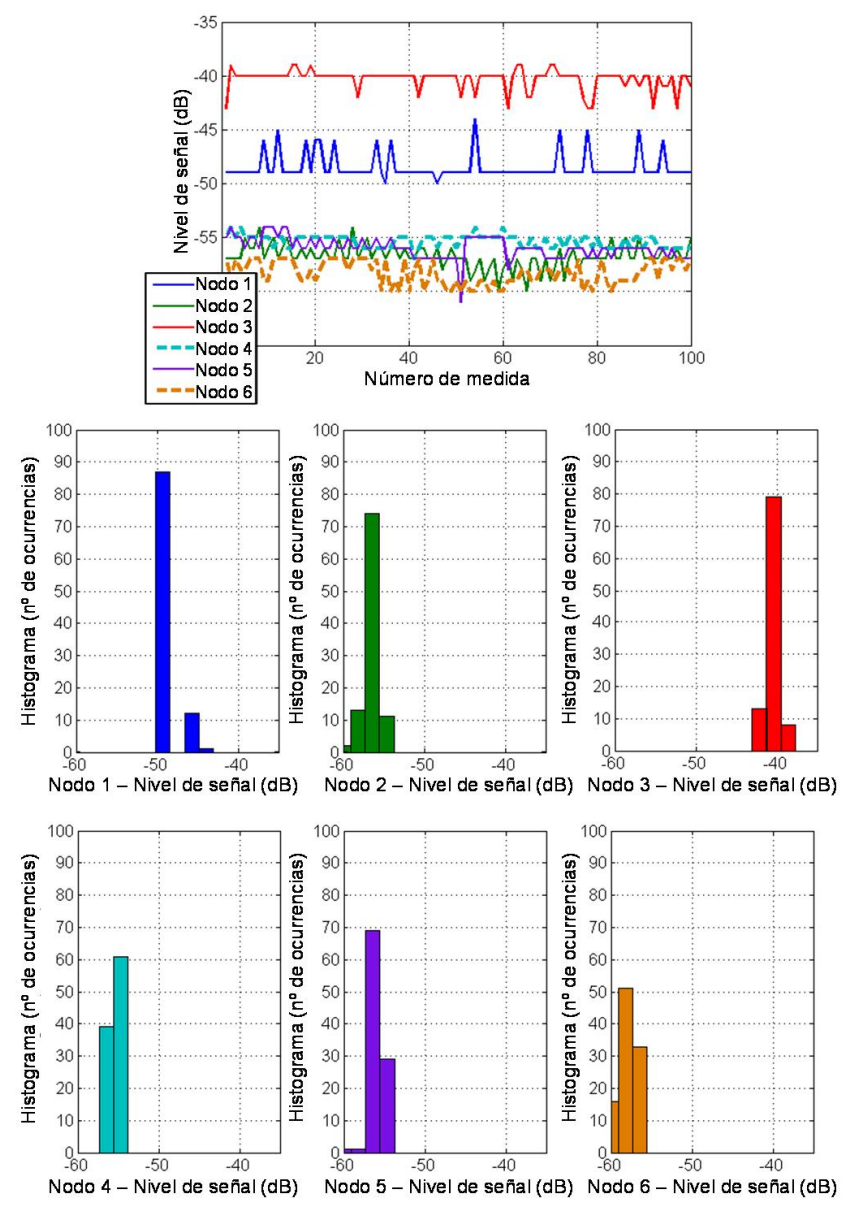

Figura 5. Niveles RSS medidos en la posición de evaluación \#1 y sus correspondientes histogramas.

La Tabla II analiza el error cometido para diverso número de muestras promediadas. Desde el punto de vista de implementación del sistema, se emplea un buffer de tipo FIFO (First In First Out), donde el tamaño del buffer corresponde al número de muestras que se desea promediar. Por ejemplo, un buffer de 10 muestras implica que la posición se proporcionará con un retardo de $5 \mathrm{~s}$. En los resultados de la Tabla II se observa que a partir de 10 muestras promediadas, el error cometido permanece invariante.

A partir de la Tabla II es posible realizar una extrapolación de la precisión proporcionada por el ILS implementado, aunque el número de posiciones de evaluación ( 5 posiciones) 
no es suficientemente significativo. Se concluye que el error máximo, $2.1 \mathrm{~m}$ (17.5\% con respecto a la diagonal de la planta de la vivienda), corresponde a la posición \#2, situada en la periferia del escenario. Asimismo, las posiciones con menor error son aquellas situadas en el centro de la vivienda, o a distancias inferiores a $2 \mathrm{~m}$ de alguno de los nodos estáticos. A partir de dicha distancia, el valor RSS puede estar próximo al nivel de sensibilidad de los nodos ZigBee, es decir, al nivel de ruido.

TABLA II

ERROR COMETIDO EN FUNCIÓN DEL NÚMERO DE MUESTRAS PROMEDIADAS

\begin{tabular}{cccccc}
\hline \hline $\begin{array}{c}\text { No. } \\
\text { muestras }\end{array}$ & POS.\#1 & POS.\#2 & POS. \#3 & POS. \#4 & POS. \#5 \\
\hline 5 & $0.5 \mathrm{~m}$ & $2.1 \mathrm{~m}$ & $1.9 \mathrm{~m}$ & $1.3 \mathrm{~m}$ & $1.3 \mathrm{~m}$ \\
10 & $0.5 \mathrm{~m}$ & $2.1 \mathrm{~m}$ & $1.8 \mathrm{~m}$ & $1.2 \mathrm{~m}$ & $1.2 \mathrm{~m}$ \\
20 & $0.5 \mathrm{~m}$ & $2.1 \mathrm{~m}$ & $1.8 \mathrm{~m}$ & $1.2 \mathrm{~m}$ & $1.2 \mathrm{~m}$ \\
\hline \hline
\end{tabular}

Otro tipo de análisis de interés en sistemas ILS es la influencia del número de nodos en la precisión. En este caso, el estudio se ha realizado desactivando diferentes nodos en el escenario, y considerando un tamaño de buffer de 10 muestras. En la Tabla III se compara el error cometido para cada una de las posiciones de evaluación cuando se tienen activos todos los nodos y cuando se desactivan nodos situados en el centro de la vivienda (el 1 y el 2). El incremento del error observado en la Tabla III es proporcional a la proximidad de la posición de evaluación a alguno de los nodos desactivados.

TABLA III

ERROR COMETIDO EN FUNCIÓN DE LOS NODOS ACTIVOS

\begin{tabular}{cccccc}
\hline \hline $\begin{array}{c}\text { Nodos } \\
\text { apagados }\end{array}$ & Pos. \#1 & Pos. \#2 & Pos. \#3 & Pos. \#4 & Pos. \#5 \\
\hline Ninguno & $0.5 \mathrm{~m}$ & $2.1 \mathrm{~m}$ & $1.8 \mathrm{~m}$ & $1.2 \mathrm{~m}$ & $1.2 \mathrm{~m}$ \\
2 & $0.7 \mathrm{~m}$ & $2.2 \mathrm{~m}$ & $2 \mathrm{~m}$ & $1.4 \mathrm{~m}$ & $1.2 \mathrm{~m}$ \\
1,2 & $0.7 \mathrm{~m}$ & $2.2 \mathrm{~m}$ & $2 \mathrm{~m}$ & $1.5 \mathrm{~m}$ & $1.3 \mathrm{~m}$ \\
\hline \hline
\end{tabular}

\section{VALIDACIÓN EXPERIMENTAL}

En esta sección se pretende demostrar la viabilidad del mismo para la localización, seguimiento de objetos y personas. El escenario seleccionado corresponde a los laboratorios del Área de Teoría de la Señal y Comunicaciones, en Gijón (Asturias), España. Debido a que el número máximo de nodos ZigBee disponibles es de 8, los 6 nodos configurados como estáticos se han distribuido a lo largo de 2 salas tal y como se muestra en la Fig. 6, intentado de nuevo maximizar la zona de cobertura. También se ha aumentado ligeramente la densidad de nodos en una sala con respecto a la otra para poder evaluar la influencia en el seguimiento de los objetos. Como se recoge en [26] es posible llevar a cabo una optimización más rigurosa de la posición de los nodos, si bien no es objeto de estudio en la presente contribución.

En este escenario, el tiempo de despliegue y puesta a punto del sistema ILS ha sido de 30 minutos, correspondiente a las operaciones de colocar, activar, mapear las coordenadas de los nodos estáticos, y creación del escenario en el ordenador donde se ejecuta el algoritmo de localización.

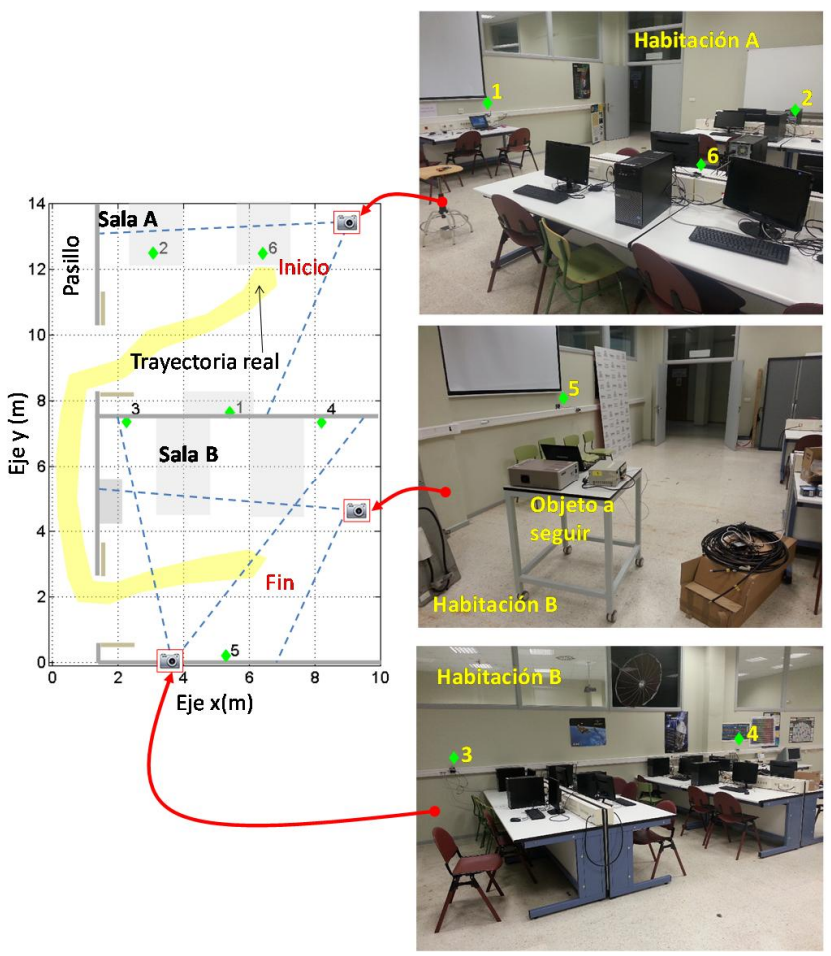

Figura 6. Escenario donde se ha desplegado el ILS. Izquierda: plano de situación. Derecha: fotografías del escenario.

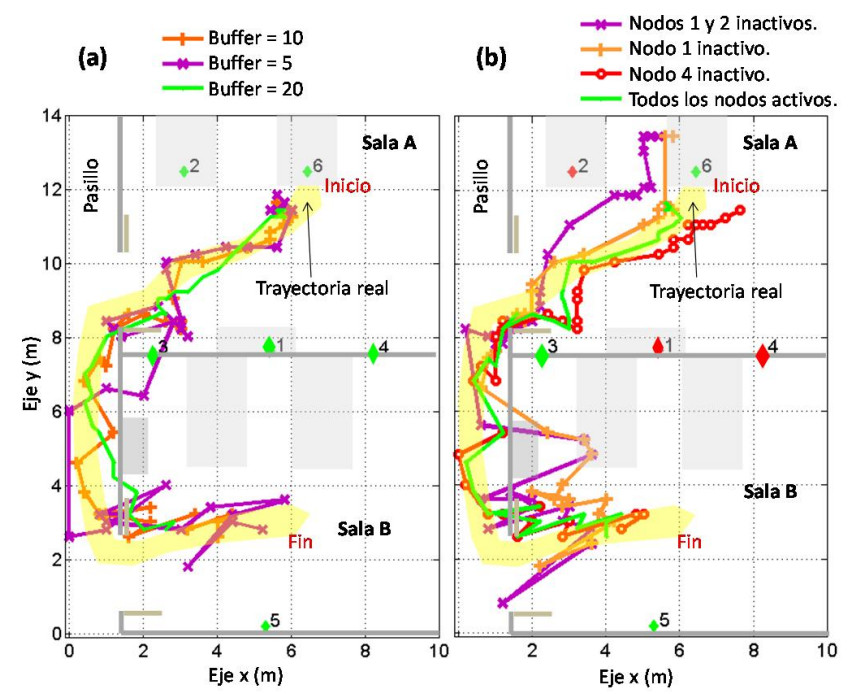

Figura 7. Seguimiento de una persona moviendo un carrito de la sala A a la B. Izquierda (a): análisis para diferentes tamaños de buffer. Derecha (b): análisis para diferentes conjuntos de nodos activos empleando un buffer de 10 muestras.

Los resultados de localización y seguimiento para una persona moviendo un carrito de la sala A a la B se muestran en la Fig. 7. Se han analizado los casos para diferentes tamaños de buffer $(5,10$, y 20 muestras) y de nodos estáticos activos. Para la configuración correspondiente a todos los nodos activos y tamaño de buffer de 10 muestras, el error máximo entre la trayectoria real de la persona y la estimada por el ILS es de $1 \mathrm{~m}$. Nuevamente el error se define como la distancia euclídea entre la posición del real del carrito (para ello, la trayectoria a seguir se ha señalizado previamente en el 
suelo) y la posición estimada por el sistema ILS.

En este ejemplo se observa también cómo el nodo 4 , que es el más alejado de la trayectoria real, no tiene un peso significativo en el error cometido, ya que las trayectorias estimadas son similares independientemente de que esté activo o no. El efecto contrario sucede para el nodo 1, situado más próximo a la trayectoria real.

Otro efecto apreciable es el de la influencia de la densidad de nodos. En la sala A, al haber más nodos, la trayectoria se estima con mayor precisión. Por el contrario, la sala B está cubierta por menos nodos, por lo que se tiene más imprecisión en la estimación de la trayectoria.

En el enlace https://goo.gl/R4Iw3U se encuentra disponible un vídeo donde se puede visualizar el cálculo de la trayectoria mientras la persona se desplaza de la sala A a la B.

\section{CONCLUSIONES}

En esta contribución se ha presentado un ILS basado en RSS implementado con una red de sensores ZigBee, capaz de proporcionar una precisión entre el 6.5 y el $17 \%$ del tamaño del escenario donde se despliega. Las ventajas que presenta el método propuesto con respecto a las técnicas descritas mencionadas en la Introducción es su rápido despliegue, ya que no es necesaria una fase previa de calibración y puesta a punto, al basarse en una función de coste que utiliza diferencias de RSS entre los nodos estáticos de la red. La validación se ha realizado en entornos reales, analizando la influencia entre el número de muestras a promediar (es decir, tiempo de actualización de la posición), y la densidad de nodos en el escenario bajo estudio.

TABLA IV

COMPARACIÓN DE ILS BASADOS EN RSS

\begin{tabular}{llll}
\hline \multicolumn{1}{c}{ Método } & $\begin{array}{c}\text { Tamaño del } \\
\text { escenario }(\mathrm{m})\end{array}$ & $\begin{array}{c}\text { Error } \\
\text { absoluto }(\mathrm{m})\end{array}$ & $\begin{array}{c}\text { Error relativo } \\
(\%)\end{array}$ \\
\hline Fingerprinting [7] & $41.5 \times 11.3$ & $1^{(1)}-3.5$ & $2.3^{(1)}-8$ \\
Ecolocation [16] & $8 \times 15$ & $0.7^{(1)}-1.7$ & $4.1^{(1)}-1.7$ \\
ZigBee [18,19] & $10 \times 6$ & $0.5^{(1)}-2$ & $4.2^{(1)}-17$ \\
LQI - ZigBee [8] & $15 \times 2$ & $0.7-1.8$ & $4.6-12$ \\
Esta contribución & $12 \times 8$ & $1-2.5$ & $6.5-17$ \\
MoteTrack [17] & $41.8 \times 41.8$ & 4 & 6.7 \\
ZigBee [11] & $1 \times 1$ & 0.1 & 7 \\
ZigBEACON [10] & $11 \times 5.75$ & $1.15^{(1)}-1.7$ & $9.3^{(1)}-13.5$ \\
LANDMARC [12] & $11 \times 5.75$ & $1.6^{(1)}-2.5$ & $13^{(1)}-20$ \\
Hybrid RFID-WSN [13] & $12 \times 10$ & $2.4^{(1)}$ & $15.6^{(1)}$ \\
Wifi, Bluetooth [15] & $23 \times 23$ & 5 & 21 \\
MLE - ZigBee [9] & $8 \times 9$ & $1.8^{(1)}$ & $26^{(1)}$ \\
Bluetooth [14] & $4.5 \times 5.5$ & 2 & 28 \\
\hline \hline
\end{tabular}

(1) Promediado.

(2) Despliegue en exteriores.

(3) Error relativo a la diagonal del escenario (D): error relativo $=100 \mathrm{x}$ error absoluto / D.

Como se desprende de la Tabla IV, la precisión del sistema propuesto está en línea con la proporcionada por otros ILS tales como [11,17]. Nótese que con respecto a métodos basados en Zigbee que requieren calibración previa $[8,18,19]$ el error relativo de posicionamiento promedio empeora solamente del $4.2 \%$ al $6.5 \%$, mientras que el error máximo se mantiene por debajo del $20 \%$.

\section{AGRADECIMIENTOS}

Esta investigación ha sido financiada por el Ministerio de Economía y Competitividad de España mediante el proyecto MIRIIEM TEC 014-54005-P, y por el Gobierno del Principado de Asturias mediante el proyecto PCTI 2013-2017 GRUPIN14-14.

\section{REFERENCIAS}

[1] D. Reyes, G. Millan, R. Osorio-Corparan, G. Lefranc, "Mobile Robot Navigation Assisted by GPS," IEEE Transactions Latin America, Vol. 13, No. 6, pp. 1915-1920. 2015.

[2] P. Ali-Rantala, L. Ukkonen, L. Sydanheimo, M. Keskilammi, M. Kivikoski, "Different kinds of walls and their effect on the attenuation of radiowaves indoors," in IEEE Antennas and Propagation Intl. Symposium, 2003, vol. 3, pp. 1020-1023, 22-27, June 2003.

[3] D. Micheli, A. Delfini, F. Santoni, F. Volpini, M. Marchetti, "Measurement of electromagnetic field attenuation by building walls in the mobile phone and satellite navigation frequency bands," IEEE Antennas and Wireless Propagation Letters, Vol. 14, pp. 698-702, 2015.

[4] G. Gracioli, R. P. Pires, L. Wanner, " Evaluation of an RSSI-based location algorithm for wireless sensor networks," IEEE Transactions Latin America, Vol. 9, No. 1, pp. 830-835, 2011.

[5] Z. Farid, R. Nordin, M. Ismail, "Recent Advances in Wireless Indoor Localization Techniques and System," Journal of Computer Networks and Communications, pp. 1-12, 2013.

[6] Y. Chu, A. Ganz, "A UWB-based 3D location system for indoor environments," Proceedings of the 2nd IEEE International Conference on Broadband Networks, BroadNets 2005. pp. 1147-1155. 2005.

[7] T. Alhmiedat, G. Samara, A. O. A. Salem, "An Indoor Fingerprinting Localization Approach for ZigBee Wireless Sensor Networks," European Journal of Scientific Res., Vol. 105, No. 2, pp. 190-202. 2013.

[8] S. J. Halder, J.-G. Park, W. Kim, "Adaptive Filtering for Indoor Localization using ZIGBEE RSSI and LQI Measurement," Adaptive Filtering Applications, INTECH publisher. pp. 305-324. InTech. 2011.

[9] H. Cho, M. Kang, J. Park, B. Park, H. Kim, "Performance analysis of location estimation algorithm in ZigBee networks using received signal strength," Proceedings of the 21st International Conference on Advanced Information Networking and Appl., Vol. 2, pp. 302-306. 2007.

[10] C. N. Huang, C. T. Chan, C. T. " ZigBee-based indoor location system by k-nearest neighbor algorithm with weighted RSSI," Procedia Computer Science, Vol. 5, pp. 58-65. 2011.

[11] G. Blumrosen, B. Hod, T. Anker, D. Dolev, B. Rubinsky, "Enhancing RSSI-based tracking accuracy in wireless sensor networks," $A C M$ Transactions on Sensor Networks, Vol. 9, No. 3, p. 29. 2013.

[12] L. M. Ni, Y. Liu, Y. C. Lau, A. P. Patil, "LANDMARC: indoor location sensing using active RFID," Wireless networks, Vol. 10, No. 6, pp. 701710. 2004.

[13] Z. Xiong, Z. Song, A. Scalera, E. Ferrera, F. Sottile, P Brizzi, "Hybrid WSN and RFID indoor positioning and tracking system," EURASIP Journal on Embedded Systems, Vol. 1, pp. 1-15, 2013.

[14] U. Bandara, M. Hasegawa, M. Inoue, H. Morikawa, T. Aoyama, "Design and implementation of a Bluetooth signal strength based location sensing system," Proceedings of 2004 IEEE Radio and Wireless Conference, pp.319-322, September 2004.

[15] A. K. Hossain, H. Nguyen Van, Y. Jin, W.-S. Soh, "Indoor Localization Using Multiple Wireless Technologies," Proc. 2007 IEEE Intl. Conference on Mobile Adhoc and Sensor Systems, pp. 1-8. 2007.

[16] K. Yedavalli, B. Krishnamachari, S. Ravula, B. Srinivasan, "Ecolocation: a sequence based technique for RF localization in wireless sensor networks," In Proceedings of the 4th international symposium on Information processing in sensor networks. IEEE press. p. 38. 2005.

[17] K. Lorincz, M. Welsh, "Motetrack: A robust, decentralized approach to RF-based location tracking. In Location-and Context-Awareness," Springer Berlin Heidelberg. pp. 63-82. 2005.

[18] Y. Álvarez, M. E. de Cos, J. Lorenzo, F. Las-Heras, F. "Novel received signal strength-based indoor location system: development and testing," EURASIP Journal on Wireless Communications and Networking, Vol. 4, pp. 1-10. 2010.

[19] Y. A. López, M. E. C. Gómez, J. L. Álvarez, F. L.-H. Andrés, "Evaluation of an RSS-based indoor location system," Sensors and Actuators A: Physical, Vol. 167, No. 1, pp. 110-116. 2011. 
[20] S. J. Orfanidis, "Electromagnetic Waves and Antennas," Ed. Rutgers University, Ch. 16, p. 754, http://www.ece.rutgers.edu/ orfanidi/ewa/ Accessed August 15, 2016.

[21] C. A. Balanis, "Antenna theory: analysis and design," Ed. John Wiley \& Sons. 2012

[22] IEEE Computer Society (2003). IEEE Std 802.15.4-2003. IEEE Std 802.15.4., Part 15.4: Wireless Medium Access Control (MAC) and Physical Layer (PHY) specification for Low Rate wireless Personal Area Networks (LR-WPANs).

[23] ZigBee Alliance. http://www.zigbee.org Accessed August 10, 2016.

[24] XBeeTM, XBee-PROTM OEM RF Modules. MaxStream. http://ftp1.digi.com/support/documentation/manual_xb_oem-rfmodules_802.15.4_v1.xAx.pdf Accessed August 16, $20 \overline{16}$.

[25] Battery Life Calculator datasheet. Digi.

ftp1.digi.com/support/utilities/batterylifecalculator.xls Accessed August 15, 2016.

[26] A. Krause, C. Guestrin, A. Gupta, J. Kleinberg, "Near-optimal sensor placements: Maximizing information while minimizing communication cost," In Proceedings of the ACM 5th international conference on Information processing in sensor networks. pp. 2-10. April 2006.

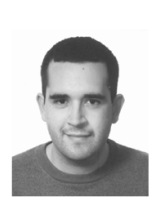

Yuri Alvarez es Doctor Ingeniero en Telecomunicación por la Universidad de Oviedo (2009). Ha realizado estancias de investigación postdoctoral en el ALERT Center of Excellence, Northeastern University, Boston, USA, de 2011 a 2014.

Actualmente es Profesor Ayudante Doctor en el Área de Teoría de la Señal y Comunicaciones (TSC-UNIOVI) de la Universidad de Oviedo (Asturias, España). Sus intereses de investigación se centran en el estudio y caracterización de antenas, técnicas de medida de antenas, aplicaciones de imaging electromagnético, y técnicas de localización en interiores mediante técnicas de radiofrecuencia.

Fernando Las Heras es Doctor Ingeniero en Telecomunicación por la Universidad Politécnica de Madrid (1990). De 1991 a 2000 fue Profesor Ayudante en dicha universidad. Desde diciembre de 2003 es Catedrático en la Universidad de Oviedo, dirigiendo el grupo de investigación TSC-UNIOVI. Ha sido autor y co-autor de más de 300 artículos y contribuciones a congresos en las temáticas de diseño de antenas, problema inverso en electromagnetismo, diagnóstico y caracterización de antenas, técnicas de recuperación de fase, imaging electromagnético en bandas milimétricas y de terahercios, localización en interiores, y metodologías docentes en ingeniería. 